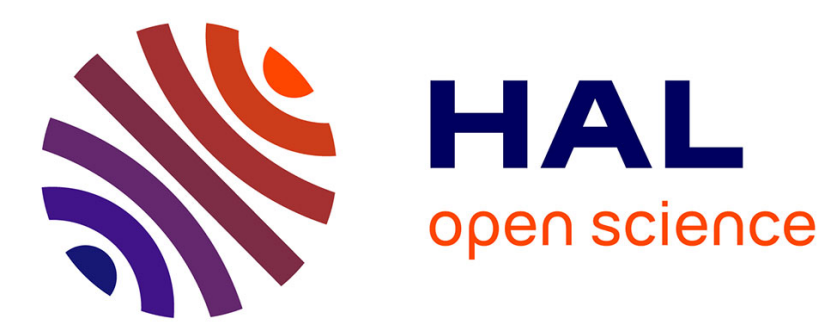

\title{
Police, justice et discriminations raciales
}

Fabien Jobard

\section{To cite this version:}

Fabien Jobard. Police, justice et discriminations raciales. D. Fassin, É. Fassin. De la question sociale à la question raciale? Représenter la société française, La Découverte, pp.211-229, 2009, 9782707149732. hal-00556749

\section{HAL Id: hal-00556749 \\ https://hal.science/hal-00556749}

Submitted on 11 May 2015

HAL is a multi-disciplinary open access archive for the deposit and dissemination of scientific research documents, whether they are published or not. The documents may come from teaching and research institutions in France or abroad, or from public or private research centers.
L'archive ouverte pluridisciplinaire HAL, est destinée au dépôt et à la diffusion de documents scientifiques de niveau recherche, publiés ou non, émanant des établissements d'enseignement et de recherche français ou étrangers, des laboratoires publics ou privés. 
Police, justice et discriminations raciales

C'est un trait commun à la France, aux États-Unis et à la Grande-Bretagne de constater, au départ de violences urbaines, un incident policier mettant en scène des policiers et des jeunes hommes de couleur, ou d'origine étrangère. Un simple regard sur les événements déclencheurs des plus graves émeutes survenues ces quinze dernières années en France est à cet égard édifiant : Aïssa Ichich, 18 ans, décédé en garde à vue, puis Youssef Khaïf, 23 ans, tué d'une balle dans la nuque (Mantes-laJolie, 1991), Makomé M’Bowole, 17 ans (Paris en 1993), Abdlekader Bouziane, 17 ans (Dammarie-lès-Lys, en 1997), Habib Ould-Mohammed, 17 ans (Toulouse, 1998), Ryad Hamlaoui, 20 ans (Lille, 2000), Mourad Belmokhtar, 17 ans (Nîmes, 2003), ou Ziad Benna, 17 ans et Banou Traoré, 15 ans (Clichy-sous-Bois, 2005).

Si la nature des institutions pénales ne se résume pas aux interventions létales de la police, la récurrence des confrontations tragiques entre policiers et fils et petit-fils d'étrangers constitue aujourd'hui un problème incontournable de la police française. L'institution judiciaire, de son côté, ne peut cacher l'évidente surreprésentation des étrangers à mesure que l'on gravit les échelons de la sévérité pénale : s'ils représentent moins de $6 \%$ de la population française, ils constituent bon an mal an $20 \%$ des personnes interpellées, $30 \%$ des personnes incarcérées dans l'année et $25 \%$ des personnes détenues. Et cette information masque les Français dits «issus de l'immigration », sur lesquels les institutions pénales (justice et police) restent aussi aveugles que l'ensemble des organismes publics.

Entretenant cette méconnaissance, le système pénal montre une cécité criante à l'égard de l'enjeu que représentent les discriminations raciales dont il est, ou serait, producteur. La présente contribution vise donc à faire la lumière sur ces

\footnotetext{
* Centre de recherches sociologiques sur le droit et les institutions pénales, CNRS
} 
discriminations. À cette fin, nous présenterons ici à la fois les travaux français existants, et des données tirées d'enquêtes (quantitatives et qualitatives) que nous avons personnellement menées.

Avant cela, précisons que ce n'est pas le racisme individuel ou institutionnel (le racisme faisant par ailleurs l'objet d'un traitement spécifique dans l'ouvrage) qui est objet de l'investigation, mais bien les pratiques, individuelles et collectives, productrices de discrimination. Les recherches sur la police française ont établi la prégnance d'attitudes racistes parmi ses agents, qui sont plus le produit de la socialisation professionnelle que celui de dispositions individuelles des policiers (selon l'adage bien connu : « dans la police, on n'est pas raciste, on le devient ») $)^{1}$. Racisme et discrimination n'entretiennent jamais un rapport de pure réciprocité : la recherche anglo-saxonne, autrement plus documentée que la nôtre, rappelle constamment que l'opinion (raciste) ne produit pas nécessairement, en situation, de comportement (discriminatoire), de même que toute discrimination n'est pas nécessairement l'agrégation de décisions individuelles à caractère raciste, mais peut résulter de la composition de divers facteurs qui, chacun, n'ont pas ou peu de lien avec le racisme ${ }^{2}$.

Travaillant sur les discriminations, nous n'évoquerons pas ce qui échoit en propre à la police des étrangers. Les logiques spécifiques de cette prérogative de l'État sont toujours susceptibles d'irradier l'action quotidienne de l'ensemble des agents, d'autant plus dans le contexte actuel, caractérisé par la fixation d'objectifs chiffrés à l'attention des préfets en matière d'éloignement du territoire français ${ }^{3}$. Ces exigences inédites de productivité peuvent imprégner l'ensemble des activités policières de plus en plus

Les policiers peuvent adhérer à l'idée que l'immigration est «la cause principale de la délinquance» (enquête par questionnaires: Dominique MONJARDET, Catherine GORGEON, La socialisation professionnelle des policiers. Etude longitudinale de la $121^{e}$ promotion des élèves gardiens de la paix. IHESI, Paris, 1993) ou bien déclarer être "devenus racistes» au contact des collègues et/ou du terrain (entretiens individuels ou collectifs : Michel WIEVIORKA et al., La France raciste. Seuil, Paris, 1992, p. 262, Dominique L'HuILliER, Les policiers au quotidien. Une psychologue dans la police. L'Harmattan, Paris, 1987, p. 121). Sur la gendarmerie, voir Renée ZAUBERMAN, "Gendarmerie et gens du voyage en région parisienne ", Cahiers internationaux de sociologie, 105, 1998, p. 415-452.

${ }^{2}$ Sur la police : Robert REINER, The politics of the police. Harvester, Londres, 1992 et la synthèse plus récente de Michael Rowe, Policing, race and racism. Willan, Cullhompton, 2004.

${ }^{3}$ Voir la circulaire conjointe du ministre de l'Intérieur et du garde des Sceaux datée du 21 février 2006 vise à assouplir les conditions d'interpellation des étrangers notamment aux guichets des préfectures. Cette circulaire fait suite à celle du 22 octobre 2003, qui imposait aux préfets des contingents annuels de reconduites à la frontière. 
saisies par l'impératif de rendement chiffré, et encourager les policiers à constater toujours plus d'infractions à la législation sur les étrangers, activité par définition discriminatoire. Nous essayerons donc ici de mettre en lumière les activités éventuellement discriminatoires imputables à la police et à la justice dans l'exercice de leurs missions ordinaires, tant que ces dernières ne sont pas entièrement assujetties aux logiques d'action propre à la police des étrangers.

Procédons par questions successives : y a-t-il discrimination dans la composition des professions judiciaires et policières ? Y a-t-il discrimination dans l'activité de la justice, puis de la police?

\section{Y a-t-il recrutement discriminatoire dans la police et la justice ?}

Sur ce point précis, nous savons peu de choses et ce alors même que les recrutements des policiers et des magistrats connaissent trois bouleversements majeurs depuis le début des années 1990 : l'augmentation des candidatures féminines et la meilleure réussite aux concours des femmes par rapport aux hommes (les promotions de l'École nationale de la magistrature sont désormais à hauteur de trois-quarts d'entre elles féminines, le ministère de la Justice ne jouant pas, comme le ministère de l'Intérieur, sur les exigences physiques au recrutement pour endiguer la féminisation de la profession) ; l'augmentation du niveau de diplôme des recrutés ; l'accent mis, du côté des décideurs politiques, sur la diversité sociale et ethnique des recrutements policiers, dont l'une des manifestations fut la création des postes d'Adjoints de sécurité (ADS).

L'expérience ADS n'a pas encore été pleinement analysée, alors même que d'autres initiatives ont récemment vu le jour, telles les «cadets de la République » ou la « classe préparatoire » au concours de commissaire (treize élèves en 2005, année de création) destinée à rompre les logiques discriminatoires à l'œuvre dans le choix des filières d'enseignement supérieur permettant l'acquisition du bagage nécessaire au concours. A ce jour toutefois, les enseignements de l'expérience ADS semblent mitigés : ayant acquis un «demi-statut» policier, ils (et elles) se sont trouvés en première ligne dans la conduite des opérations dévalorisées, notamment lors de la mise en place précipitée des antennes de police de proximité dans les cités, concentrant 
alors sur leur personne les récriminations de jeunes qu'ils étaient pourtant appelés à «mieux représenter » au sein des forces de police ${ }^{4}$.

La seule recherche dont on dispose porte sur deux concours de la Police nationale (Lille et Marseille, concours gardien de la paix 1999, concours ADS 1998). Elle a établi une part faible mais non négligeable de candidatures maghrébines ${ }^{6}(6,5 \%$ à Lille, $11 \%$ à Marseille pour les concours de gardiens, $8,3 \%$ et $17 \%$ pour les concours ADS), mais un taux de réussite inférieur de plus de trois points des Maghrébins au concours de gardien de la paix ( $12 \%$ contre $8,5 \%$ ). L'écart est particulièrement fort à Marseille, où, toutes choses égales par ailleurs, le risque d'échec des candidats maghrébins au concours de gardien est trois fois plus élevé que le risque d'échec des autres candidats. L'examen oral est en effet privilégié à Marseille, où les chercheurs ont observé que les policiers examinateurs se montraient particulièrement redoutables dans le face-à-face avec les candidats maghrébins, notamment ceux en provenance des quartiers périphériques ${ }^{7}$. Le fameux «oral» des concours de la fonction publique permet ainsi de refouler les candidats maghrébins à l'entrée de la Police nationale, comme le grand oral de l'ENA les candidats de basse extraction sociale. Ce mode de recrutement permet ainsi des pratiques discriminatoires abritées par le secret des délibérés, alors que la vigilance du Conseil de l'Europe en matière d'égal accès des hommes et des femmes à la fonction publique a par exemple, dans le même temps, obligé l'institution policière à déployer des trésors de ruse pour limiter l'entrée des femmes dans la police ${ }^{8}$.

\footnotetext{
${ }^{4}$ Renée Zauberman, René LÉVY, « Police, minorities, and the French ideal”, Criminology, 41, 4, 2003, p. 1065-1100.

5 Dominique DUPREZ, Michel PINET, Policiers et médiateurs. Sur le recrutement et les appartenances. CLERSE, Lille, 2001 (disponible en ligne sur http://www.univ-lille1.fr/clerse). Ces données convergent avec celles du journaliste Piotr SMOLAR qui a relevé sur les listes de promus des écoles de gardiens de la paix que $7 \%$ des élèves portent un nom à consonance maghrébine ou africaine en 2005-2006, contre 4,4\% en 2001-2002 et moins de 2\% en 1995 ( « Une lente amélioration dans la police ", Le Monde, 23 janvier 2006).

${ }^{6}$ Identifiées de manière patronymique depuis les dossiers de candidatures.

${ }^{7}$ Les candidats maghrébins devaient alors subir le feu de questions telles que : «si ton cousin menace un de tes collègues, sors-tu ton arme ? ", "si tu dois arrêter ton frère, que fais-tu ? ", qui ne laissent pas de place au doute quant à l'articulation entre perceptions stéréotypées et mécanique discriminatoire (ce type de questions n'était pas posé aux autres candidats, qui se trouvaient donc évalués sur d'autres critères).

${ }^{8}$ Après la suppression, en 1992, du contingentement du nombre de femmes admises au concours, le ministère de l'Intérieur a relevé la taille exigée des candidates, puis les coefficients des épreuves
} 
Didier Fassin, Eric Fassin (dir.), De la question sociale à la question raciale ?Représenter la société française. Paris : La Découverte, 2006, p. 211-229.

\section{Les décisions pénales sont-elles discriminatoires?}

Certains pays étrangers disposent de données considérables sur la question du «sentencing », l'étude des déterminants des peines prononcées par les juges ${ }^{9}$. En France, nous disposons sur ce chapitre d'une connaissance ridiculement éparse. La Cimade, une association importante d'aide aux étrangers sous le coup d'une mesure d'éloignement, a récemment livré des données à la fois tranchées et fragiles ${ }^{10}$. Seize bénévoles ont observé, de mars à juin 2002, 382 audiences correctionnelles au Tribunal de grande instance (TGI) de Montpellier, distinguant les 480 prévenus selon leur nationalité. Après avoir constaté une surreprésentation flagrante des étrangers parmi les prévenus (un comparant sur quatre), ils ont montré une différence notable de sévérité des peines prononcées: si $30 \%$ des Français jugés écopent d'un emprisonnement ferme, la proportion s'élève à $43 \%$ chez les étrangers. Et cet écart ne semble pas imputable à une différence de profils judiciaires : parmi les prévenus inscrits au casier judiciaire, ceux condamnés à une peine d'emprisonnement ferme constituent $47 \%$ de la population chez les étrangers, et $30 \%$ de la population chez les Français ${ }^{11}$.

Cette recherche a curieusement retenu la distinction Français/étrangers pour caractériser les prévenus; alors même que les données sont tirées d'observations d'audiences. L'étude documente ce que l'on sait depuis longtemps (et qui n'est pas propre à la France): un étranger est plus fréquemment condamné à une peine d'emprisonnement ferme sur la base du « défaut de garanties de représentation ». Les juges désignent par là le fait que l'étranger condamné à une amende est suspecté de se

sportives, et finalement introduit tout récemment l'indice de masse corporelle, défavorable aux femmes (Geneviève PRUVOST, L'accès des femmes à la violence légale. La féminisation de la police (1935-2005). EHESS, Paris, 2006, p. 261-264 et 433-450).

${ }^{9}$ La recherche la plus exhaustive sur cette question étant, en Europe, celle de Roger HoOD, Race and sentencing. A study in the Crown Court. Report for the commission for racial equality, Clarendon Press, Oxford, 1992, qui montre à partir d'une base constituée de 2884 décisions judiciaires que la variable raciale, parce qu'elle est porteuse d'autres variables prédictives (par exemple la récidive ou le choix de la procédure de plaider-coupable), est déterminante de la sévérité des peines prononcées.

${ }^{10}$ « La misère des prétoires », hors-série de Causes communes, janvier 2004.

${ }^{11}$ Et les proportions sont les mêmes chez les personnes jamais condamnées (ou amnistiées) : $38 \%$ des étrangers sans inscription au casier judiciaire sont condamnés, contre $25 \%$ des Français sans inscription. 
Didier Fassin, Eric Fassin (dir.), De la question sociale à la question raciale ?Représenter la société française. Paris : La Découverte, 2006, p. 211-229. soustraire à l'exécution de sa peine en regagnant son pays d'origine ${ }^{12}$. L'emprisonnement est donc systématiquement préféré à l'amende. Si les observateurs de la Cimade avaient relevé non pas l'appartenance nationale, mais l'apparence, les écarts auraient sans doute été (toutes choses égales par ailleurs) plus réduits.

Intéressé pour notre part aux « infractions à personnes dépositaires de l'autorité publique $»(\text { IPDAP })^{13}$, nous avons collecté et traité un échantillon de plus de 1800 affaires d'outrage, de rébellion et violence à agent de la force publique jugées sur un TGI de la grande banlieue parisienne, afin notamment de déterminer si les peines prononcées se distinguent selon les groupes de prévenus, constitués selon leurs patronymes et/ou lieux de naissance en trois groupes qui reprennent les «types» utilisés par les policiers («type européen », « type nord-africain », « type africain »). Sur la période la plus récente (1995-2003), on compte ainsi environ $25 \%$ de prévenus relevant du groupe «nord-africain » (nés au Maghreb ou portant un nom ou prénom maghrébin) et $20 \%$ relevant d'un groupe « africain » (nés en Afrique ou aux DOMTOM, ou portant un nom ou prénom à consonance africaine $)^{14}$. Chez les mineurs jugés, les proportions s'élèvent respectivement à $38 \%$ et $28 \%$ de l'échantillon.

Il existe une différence significative de décisions rendues selon les groupes d'appartenance: si les peines d'emprisonnement ferme sanctionnent le quart des prévenus du groupe «nord-africain », elles ne frappent qu'un peu plus du dixième seulement des prévenus du groupe «européen »; la durée moyenne des peines d'emprisonnement ferme prononcées est de 2,6 mois pour les prévenus du premier groupe, et de 2,1 mois pour ceux du dernier - des écarts considérables.

Pourtant, ce que l'analyse des composantes de cet écart révèle, c'est qu'il est

12 Pierre TOURNIER, Philippe ROBERT, Etrangers et délinquances. Les chiffres du débat. L'Harmattan, Paris, 1991.

${ }^{13}$ Quand les policiers vont au Tribunal. Analyse d'un échantillon de jugements rendus en matière d'infraction à personnes dépositaires de l'autorité publique dans un TGI parisien (1965-2003), CESDIP, Guyancourt, «Etudes et données pénales », n 97 (en ligne sur www.cesdip.com). Les données évoquées ici sont les 661 IPDAP de prévenus majeurs sans infraction jointe.

${ }^{14}$ Le document duquel les données ont été tirées est le feuilleton d'audience, qui ne mentionne pas la nationalité des prévenus. Notre codage pose bien entendu de nombreux problèmes, parmi lesquels (entre autres) le versement dans le groupe «nord-africain » des noms à consonance arabe de personnes d'origine sub-saharienne nées en France, mais surtout le versement dans le groupe « européen » de tous les patronymes typiquement français de personnes nées en Métropole de parents africains, guadeloupéens ou martiniquais (Thierry Henry, footballeur français né aux Ulis (Essonne), relèverait ainsi du groupe « européen »). 
largement imputable à des facteurs strictement judiciaires. Ainsi, les prévenus du groupe «nord-africain» sont plus fréquemment que les autres jugés pour des infractions de violence à agent que pour des infractions d'outrage simple, les premières impliquant un risque d'emprisonnement ferme trois fois supérieur aux secondes ${ }^{15}$. Cette différence est un déterminant majeur de l'écart. Ensuite, il faut noter que les prévenus de ce même groupe sont plus susceptibles que ceux du groupe « européen » de ne pas se présenter à l'audience alors qu'ils ont reçu une convocation en bonne et due forme (le jugement est alors « réputé contradictoire»), appelant une peine plus lourde de la part des juges. Enfin, ce sont ces prévenus du groupe «nordafricain » qui sont surreprésentés parmi les jugés en état de récidive, suscitant mécaniquement des peines plus lourdes, la procédure pénale posant que l'état de récidive élève alors par deux le plafond de la peine maximale prévue.

En ce qui concerne la part résiduelle de l'écart, une fois identifié ce qui est imputable aux facteurs judiciaires, on sait que de manière générale les juges se retiennent de prononcer des peines d'emprisonnement ferme à l'égard des prévenus disposant d'un emploi, pour ne pas ajouter à la peine judiciaire l'exclusion durable du marché du travail ${ }^{16}$. Or, les enquêtes disponibles montrent une discrimination constante des populations d'origine étrangère dans l'accès à l'emploi ${ }^{17}$. Enregistrant ces discriminations, la justice en accroît les effets par sa logique propre, l'emprisonnement. La justice, dit-on, est aveugle... et plus encore, peut-être, à l'égard des effets discriminatoires de ses intentions vertueuses ${ }^{18}$.

Revenons toutefois sur les facteurs principaux, ces «facteurs judiciaires » que sont les types de délits, les modes de jugement et la récidive. L'étude que nous avons menée ne porte que sur la phase de l'audience pénale, et ignore ainsi tout ce qui a

${ }^{15}$ Le risque de voir une peine d'emprisonnement ferme prononcée est de $10 \%$ lorsque l'affaire jugée est un simple outrage, $20 \%$ lorsqu'il s'agit d'une rébellion, 30\% lorsqu'il s'agit d'une violence.

${ }_{16}$ Les recherches en attestent en France depuis Nicolas HERPIN, L'application de la loi. Deux poids, deux mesures. Le Seuil, Paris, 1977 et Bruno AUBUSSON DE CAVARLAY, « Hommes, peines et infractions. La légalité de l'inégalité », L'Année Sociologique, 35, 1985, p. 275-309.

${ }^{17}$ Tout récemment encore, voir Roxane SILBERMAN, Irène FOURNIER, « Les secondes générations sur le marché du travail en France. Une pénalité ethnique ancrée dans le temps », Revue française de sociologie, 47, 2, 2006, p. 243-292.

${ }^{18}$ Sur les effets discriminatoires des décisions judiciaires, Michael TONRY, Thinking about crime. Sense and sensibility in American penal culture. Oxford University Press, Oxford, 2004, p. 221-225. 
précédé, et notamment l'échange avec les policiers et la manière dont ces derniers exercent leur pouvoir de transformation d'un événement (ou non-événement) en fait judiciaire (outrage ou rébellion? transmission à l'OPJ ou non ? puis au Parquet ? etc.). Car ce qui ressort de l'examen des audiences est que la probabilité que des policiers se constituent partie civile au titre du préjudice moral est significativement plus élevée lorsque la personne poursuivie relève du groupe «nord-africain » que lorsqu'elle relève du groupe « européen ». Cet élément, qui montre un traitement indubitablement différencié des policiers à l'égard des interpellés maghrébins, impose de resserrer l'attention sur le maillon policier de la chaîne pénale.

C'est le travail auquel s'était livré René Lévy, auteur d'une étude pionnière (et malheureusement restée orpheline) sur le devenir des interpellations opérées par les brigades de voie publique en 1979-1981 ${ }^{19}$. Retenant les «types» policiers pour l'analyse de 538 dossiers d'interpellés sur la voie publique, il montrait la part décisive, dans la décision de déférer, de l'appartenance au groupe «nord-africain », par rapport à l'appartenance au groupe « européen ». La variable " groupe nord-africain » surdéterminait toutes les autres, y compris celles relatives aux garanties de représentation (nationalité, situation familiale, emploi).

Ayant de surcroît observé le travail policier sur le terrain, il avait également été amené à constater une surreprésentation des «Nord-Africains» au stade de l'interpellation, et concluait ainsi : «Dans sa composition ethnique, la population déférée n'est pas identique à la population mise en cause par la police. Et de même, cette dernière se distingue de ce point de vue de la population d'ensemble au sein de laquelle elle est prélevée. La cause de ces différences réside dans les pratiques policières sélectives qui sont mises en œuvre tant au stade de la prise en charge des affaires et des personnes, qu'au stade des décisions cruciales prises ultérieurement » (p. 145).

Le système pénal produit ainsi de manière indubitable des discriminations selon l'origine des personnes traitées. Ces discriminations semblent s'accentuer à mesure que l'on resserre l'attention autour des premières phases du traitement pénal, celles où

${ }^{19}$ René LEVY, Du suspect au coupable. Le travail de police judiciaire, Klinksieck/Médecine et hygiène, Paris/Genève, 1985. 
Didier Fassin, Eric Fassin (dir.), De la question sociale à la question raciale ?Représenter la société française. Paris : La Découverte, 2006, p. 211-229.

interviennent les décisions du Parquet et, surtout, les décisions policières. Ce sont donc les rapports avec la police qu'il faut examiner plus attentivement.

\section{Les interventions policières sont-elles discriminatoires?}

Nous voudrions confronter ces données quantitatives à quelques enseignements tirés de 200 à 250 heures d'observation du travail policier effectuées de mi-mars à mijuillet 2004 dans deux circonscriptions de police de la grande banlieue parisienne ${ }^{20}$. Nous exposerons les trois sources de discrimination souvent évoquées à propos du travail policier : selon les apparences raciales, selon les comportements des personnes contrôlées, selon les territoires d'intervention.

\section{$\underline{\text { La discrimination selon les origines }}$}

L'étude menée en 2004 par Catherine Withol de Wenden à partir des dossiers de la Commission nationale de déontologie de la sécurité est récemment venue documenter la question des discriminations policières ${ }^{21}$. Constatant que 36 dossiers sur les 78 que cette commission avait alors traités (hors Police aux frontières) émanaient de personnes « étrangères ou d'origine étrangère » (avec « un fort noyau de jeunes issus de l'immigration maghrébine de 18 à 35 ans, interpellés dans les banlieues défavorisées de la région parisienne », soit 16 requérants maghrébins), l'auteur concluait à la « surreprésentation » des Maghrébins dans les victimes d'abus policiers. Surreprésentation par rapport à quelle population ? En l'absence de données de population générale embrassant l'origine des personnes, il faudrait soumettre la «surreprésentation » invoquée à la population formée des personnes interpellées, ou gardées à vue, ou bien encore déférées par la police ${ }^{22}$.

C'est ce que des recherches anglaises ont cherché à mettre en lumière. A partir de collectes considérables d'observations de patrouilles de rue, elles ont montré que s'il y a surreprésentation indéniable des Noirs et Indo-Pakistanais contrôlés par la police

\footnotetext{
${ }^{20}$ Cette recherche s'est déroulée dans le cadre de l'ACI « Travail dans la fonction publique » (voir Marc LORIOL (dir.), Construction du stress, psychologisation du social et rapport au public. Les cas des infirmières hospitalières, des conducteurs de bus et des policiers. Laboratoire Georges Friedmann, Bourg-la-Reine, décembre 2004.

${ }^{21}$ Commission nationale de déontologie de la sécurité, Rapport annuel. La Documentation française, Paris, 2004.

${ }^{22}$ Comparons par exemple le ratio 36/78 (étude de Wenden) avec les prévenus d'IPDAP de notre propre étude: les proportions sont à peu près identiques, il n'y a pas « surreprésentation »...
} 
Didier Fassin, Eric Fassin (dir.), De la question sociale à la question raciale ?Représenter la société française. Paris : La Découverte, 2006, p. 211-229.

(«stop and search»), il n'est pas de surreprésentation observable au regard des personnes effectivement croisées par les policiers au cours de leur service ${ }^{23}$.

Pour ma part, à partir des observations que j'ai accumulées en 2004, rien ne permet d'affirmer qu'il y ait biais racial dans le choix que font les policiers de se porter vers tel ou tel individu ou groupe d'individus, et ce indépendamment des marques de racisme qui peuvent çà et là être observées (on en verra plus loin). Cet élément tranche de manière évidente avec les diverses collectes de témoignages sur les « contrôles au faciès ». C'est le paradoxe d'une enquête menée dans des banlieues difficiles : là, ce n'est pas tant la sélection des populations contrôlées par les policiers qui est en jeu, que le traitement différencié des personnes lorsqu'elles sont contrôlées ${ }^{24}$.

Cet élément exige d'examiner la nature des interventions policières et de chercher à comprendre si les personnes, lorsqu'elles sont contrôlées, interpellées ou retenues au poste, sont exposées à un traitement discriminatoire.

\section{La discrimination selon les comportements}

Dans un texte fameux de la sociologie américaine, l'ethnologue John Van Maanen avait observé la scène suivante ${ }^{25}$. Au cours d'un contrôle routier, des policiers de la « Highway Patrol » arrêtent un individu au volant de sa voiture et lui demandent ses papiers. Le conducteur fait part de son énervement aux agents : «Pourquoi donc vous m'emmerdez à me contrôler, au lieu d'arrêter les vrais délinquants? ». «Parce que vous êtes un branleur (asshole), voilà pourquoi. Mais ça, bien sûr, il a fallu que vous ouvriez votre gueule pour que je le sache ».

Van Maanen montrait ainsi que les policiers distinguent trois sortes de gens. Les « suspicious persons » sont les bons clients, qui ont commis un vrai délit et peuvent

\footnotetext{
${ }^{23}$ Voir le bilan dressé par Michael Rowe, Policing, race and racism, op. cit., p. 78-98, ainsi que le très utile Peter WADDINGTON, «Police (canteen) subculture. An appreciation », British Journal of Criminology, 39, 2, 1999, p. 287-309.

${ }^{24}$ L'étude devrait porter alors sur les équipages policiers patrouillant dans des zones où ils ne connaissent pas les populations, par exemple dans le cadre des opérations Vigipirate, dans les zones aéroportuaires, les gares, les stations de métro, zones privilégiées de contrôle préventif. Le problème est qu'ils sont alors juridiquement habilités à opérer un type de contrôle discriminatoire, sur ces zones, dans le cadre de l'article 78-2 du Code de procédure pénale, petit monument de flou juridique habilitant les policiers à faire ce que la loi ou, du moins, le Conseil constitutionnel, réprouve (cf. Fabien JOBARD, Bavures policières? La force publique et ses usages. La Découverte, Paris, 2002, p. 184-194).

${ }^{25}$ John Van MaAnen, "The asshole”, in Peter Manning et John Van MaAnen (dir.), Policing. A view from the street. Goodyear Publishing Company, Santa Monica, 1978, p. 221-238.
} 
« rapporter gros ». Ils sont rares. Les « know-nothings », l'écrasante majorité, ne sont aux yeux des policiers d'aucune utilité, ne savent rien du travail policier et n'en sauront jamais rien, bien qu'ils aient toujours un avis arrêté sur les questions de police. Enfin, les «assholes» sont tous ceux qui ne se plient pas aux attentes des policiers (par exemple, rester calme et respectueux lorsqu'ils se font contrôler) et méritent de ce fait un traitement différencié, que Van Maanen appelle la «street justice», autrement dit une bonne bastonnade.

On retrouve dans le vocabulaire des policiers que j'ai suivis cette distinction ternaire, par exemple entre les « clients », les « prouts-prouts » et les « crapauds », ces derniers toujours prompts à opposer un «quoâ? quoâ? » aux sollicitations des policiers. Toute la question est de déterminer si ces catégories sont fixées a priori, et superposables à l'apparence raciale, ou bien si elles sont le résultat du déroulement concret de l'interaction policière. Un exemple d'intervention invite à suivre résolument l'hypothèse de Van Maanen.

J'embarque à $17 \mathrm{~h} 00$ à bord d'un véhicule sérigraphié avec un équipage en tenue, formé de deux gardiens de la paix (chacun trois-quatre ans de maison), et d'une Adjointe de sécurité (ADS). Peu après, à la périphérie de la ville, nous repérons un véhicule jeune conducteur s'engageant sur un chemin de terre longeant la voie ferrée, indice qu'il s'agit là peut-être de gitans s'affairant à quelque chose qui mérite la curiosité policière.

L'équipage parvient rapidement à la hauteur du véhicule et repère au passage que les deux passagers avant n'ont pas attaché leur ceinture de sécurité. La voiture est arrêtée, et trois jeunes Maghrébins (dont une fille) en descendent, extrêmement avenants. Les policiers, visages fermés, rassemblent les papiers du conducteur, des passagers et du véhicule, et inspectent l'extérieur du véhicule en question, pour constater deux pneus lisses, le feu avant droit et le clignotant avant gauche défaillants. Froids, sévères, les deux policiers annoncent qu'il leur faudra immobiliser le véhicule. L'un des deux déclare à son collègue qu'il doit aller chercher la souche des timbres-amendes dans le coffre arrière, appelant ainsi discrètement son collègue à le rejoindre (chacun sait que la souche ne se trouve pas dans le coffre arrière).

A l'arrière du véhicule de police, il soulève le capot et attend quelques instants. Son collègue le rejoint et les deux évaluent la qualité des jeunes contrôlés. "Ils sont sympas, non ? ». "C'est sûr, ils sont pas méchants ». Une fois les protagonistes ainsi labellisés, les deux policiers s'accordent à ne constater qu'un défaut de ceinture passager, et le feu avant droit. Ils retournent vers les jeunes, cette fois souriants et blagueurs, et leur disent que, comme ils sont "polis », ils n'immobilisent pas le véhicule et ne dressent que deux PV d'infraction. Chauds remerciements, et l'équipage repart, non sans indiquer aux deux jeunes que, s'ils étaient eux, ils éviteraient de rouler dans un véhicule si peu sûr.

Journal de terrain, 16 mars 2004.

On voit bien à l'aune de cet exemple que le cadrage de la rencontre préside à la nature des échanges entre les protagonistes : le conciliabule à l'abri du capot arrière porte sur la qualification des personnes et décide du comportement qu'il convient alors 
Didier Fassin, Eric Fassin (dir.), De la question sociale à la question raciale ?Représenter la société française. Paris : La Découverte, 2006, p. 211-229.

d'adopter. On voit alors bien comment, pour comprendre le comportement discriminatoire des policiers, il est nécessaire d'examiner les opérations changeantes, itératives, négociées, de définition de la situation et des rôles de chacun.

Bien entendu, cette nécessité d'en appeler à une sociologie dynamique des situations pour envisager la question des discriminations présente le redoutable désavantage de n'être généralisable qu'au prix d'un nombre très élevé d'observations. Dans l'exemple que nous venons de citer, l'écart entre les personnes définies par leur statut et les personnes dans et par l'interaction est rendu plus visible encore par le fait que, quelques jours après, avec d'autres policiers, j'ai retrouvé le même conducteur du véhicule, cette fois passager d'une voiture conduite par un mineur. Lors de cet épisode, alors que la même personne (toujours jeune, toujours maghrébin, toujours portant casquette...) était abordée par les policiers, le ton était autrement plus rugueux.

On peut s'inquiéter de ce constat empirique, qui montre la faculté laissée à la discrétion des agents de décider du sort qu'ils réservent aux personnes, selon la logique de partition ternaire des publics, à la Van Maanen. Mais 1'on se bornerait alors à constater le caractère éminemment discrétionnaire de l'intervention policière, attesté depuis des décennies par la sociologie de la police, qui veut en effet que les policiers jouissent d'un pouvoir certain d'appréciation des situations et des réponses à leur opposer $^{26}$. Ce qu'il faudrait plutôt concevoir serait une méthode visant à repérer s'il existe chez les policiers des attentes différenciées de déférence selon les groupes rencontrés; autrement dit si, précisément lorsqu'ils sont des jeunes Maghrébins portant casquette sur un chemin de terre, les contrôlés ne sont pas de facto soumis par les policiers à une exigence plus élevée de déférence.

Une discrimination serait alors mise en évidence, qui porterait moins sur un écart entre les comportements policiers selon les groupes que sur un écart entre les comportements exigés par les policiers selon les groupes contrôlés. On retrouverait un constat assez commun des études sur les discriminations portant sur l'école ou l'emploi : l'exigence de conformité ou d'excellence est décuplée à l'égard des

\footnotetext{
${ }^{26}$ Dominique Monjardet, Ce que fait la police. Sociologie de la force publique. La Découverte, Paris, 1996, p. 37-62.
} 
Didier Fassin, Eric Fassin (dir.), De la question sociale à la question raciale ?Représenter la société française. Paris : La Découverte, 2006, p. 211-229. minorités ${ }^{27}$. L'exigence discriminatoire de déférence serait d'autant plus durement ressentie par les populations concernées que, parfaitement invisible, elle est difficilement dicible dans l'espace public. Et, comme elle ne recoupe pas exactement les catégories déontologiques (une attente différenciée de déférence est-elle constitutive d'une faute ?....), elle devient tout aussi difficilement blâmable ${ }^{28}$, ce qui décuple à la fois l'impuissance à la dénoncer, et la violence à la subir.

\section{La discrimination selon les territoires}

La rhétorique guerrière des ministres de l'Intérieur successifs depuis au moins 1993, les témoignages des populations concernées et des encadrants sociaux ou la (rare) littérature produite par les policiers eux-mêmes suggèrent que le travail policier se distingue dans les zones urbaines sensibles en ce qu'il y serait brutal et discriminatoire. Cette approche tranche en partie avec la précédente : soit l'objet de la discrimination est la personne, définie par ses origines, et l'on peut alors construire une protestation fondée sur la dénonciation de pratiques administratives et policières héritées des colonies françaises ; soit l'objet de la discrimination est le territoire, et la contestation désigne plutôt les inégalités sociales, dont la police aurait pour fonction de garantir la perpétuation. La ségrégation raciale se superposerait alors à la discrimination sociale.

La variable territoriale ne détermine sans doute pas à elle seule le comportement policier $^{29}$. Le facteur territorial ne saurait être distingué de la variable individuelle, et plus précisément de la qualité pénale de la personne. Plus que l'un ou l'autre, c'est la combinaison de ces deux facteurs qui produit le comportement policier. L'exemple suivant l'illustre assez bien.

Peu après une heure du matin, en voiture banalisée avec un équipage BAC formé de trois gardiens de la paix expérimentés (Franck, Philippe et Mathieu) ${ }^{30}$. Prenons en chasse une Opel Corsa qui monte à vive allure vers le Plateau [la cité de grands ensembles de la circonscription, marquée un an et demi avant mes observations par des affaires médiatisées de brutalités policières et des épisodes de violences collectives]. Arrivés au

\footnotetext{
${ }^{27}$ On retrouve ici ce que l'oral des examens de recrutement aux concours de gardiens de la paix mettait en lumière.

${ }^{28}$ Les conditions nécessaires à l'expression publique du préjudice sont ainsi difficilement réunies (cf. William Festinger et al., "The emergence and transformation of disputes. Naming, blaming, claiming », Law and Society Review, XV, 3-4, p. 631-654).

${ }^{29}$ Fabien JOBARD, «Sociologie politique de la racaille. Les formes de passage au politique des 'jeunes bien connus des services de police' », in Hugues LAGRANGE, Marco OBERTI (dir.), Emeutes urbaines et protestations. Une singularité française. Paris : Presses de Sciences-po, 2006, p. 59-79.

${ }^{30}$ Patronymes, prénoms ou noms de lieux cités dans les extraits de journaux de terrain sont fictifs.
} 
Plateau, alors que l'Opel Corsa n'est de toutes façons plus en vue, notre voiture pile à la hauteur de cinq ou six jeunes Maghrébins d'une bonne vingtaine d'années. Les policiers en sortent très vite et leur demandent leurs papiers d'identité, tout en les palpant de manière assez superficielle. Les jeunes en question n'ouvrent absolument pas la bouche, restent d'une placidité frisant la nonchalance, dont ils ne font pas non plus démonstration ostentatoire. Philippe leur rend très vite leurs papiers en leur disant, un brin impressionné par l'absence d'état de service des contrôlés : "Vous êtes du Plateau et on ne vous a jamais vus, c'est bien! Ah! Non, vraiment, si tout le monde était comme vous, je vous assure, on aurait moins de boulot. " Remontant en voiture, je lui demande la raison du contrôle, n'ayant moi-même absolument rien repéré à la hauteur des jeunes. Il me dit qu'il lui avait semblé voir deux d'entre eux rebrousser chemin à notre arrivée. Quelques minutes après, alors que nous nous trouvons à un feu rouge, le passager d'une voiture nous passant devant à vive allure nous salue d'un geste franc. Mathieu s'écrie alors : «C'est Rachid Allouache qui conduisait! Putain, il ne lui reste plus que trois points sur son permis. Je veux être celui qui lui fera perdre son permis! ». Mais Franck ne prend pas la voiture en chasse.

Journal de terrain, 15 mai 2004.

Selon toute évidence, le territoire est un marqueur fort du comportement policier. A peine arrivé dans la cité, il suffit d'un indice absolument évanescent de comportement suspect pour déclencher le contrôle. Lequel, exécuté sur le territoire policier, ressort moins de la pure démonstration de force que de la mise à l'épreuve de la qualité des personnes. En l'espèce, constatant que les jeunes, dont les papiers indiquaient qu'ils étaient du lieu, étaient «inconnus », les policiers ont très vite considéré que si le territoire était le leur, les jeunes n'étaient pas de leur « clientèle ». -Territoire et qualité pénale se corrigent l'un l'autre aux yeux des policiers intervenant. Le territoire autorise à lui seul l'interpellation, mais la virginité pénale dissuade le traitement préjudiciel. De même que, dans la chora de Platon, la coque de la tortue est à la fois le territoire de la tortue et son être, l'être emportant partout où il se rend un morceau de son identité (son identifiant territorial) ${ }^{31}$, le territoire est indissociable pour les policiers de la qualité pénale de ceux qui s'y trouvent. L'exemple suivant en témoigne plus précisément encore.

Les fonctionnaires BAC (Jean, Alain et trois autres, tous gardiens de la paix) sont rassemblés dans le petit local qui sert de cantine de fortune, dans le vieux commissariat, chacun attablé à sa gamelle. Le chef de poste vient nous voir et nous signale des « appels 17 » pour des perturbateurs au pied de la tour Balzac. Jean, interrompu dans son dîner, éconduit le chef de poste en lui disant qu'il revient aux équipages en tenue de prendre en charge ce type d'événements mineurs. Mais il se ravise peu après, demandant à la cantonade si ce n'est pas un certain Genzoudi qui, récemment sorti [de prison] serait revenu [tour Balzac] et y mettrait à nouveau «le bordel». Florent me dira un peu plus

31 Fabien JOBARD, „Der Ort der Politik. Politische Mobilisierung zwischen Aufstandsversuchung und Staatsgewalt in einer Pariser Vorstadt“, Berliner Journal für Soziologie, 3, 2004, p. 319-338. 
tard en effet que Genzoudi est un «bâtard», une « vermine », un « voleur » qui avait mis toute la paisible cité Balzac sens dessus dessous en y désossant toutes les voitures pour les revendre en pièces détachées et en y organisant, par ailleurs, un trafic d'essence filoutée. Il revendait même l'essence qu'il volait au garage de son propre père, ce pour quoi on ne pouvait le condamner (les délits intrafamiliaux de ce type ne pouvant faire l'objet de poursuites).

Les autres policiers, y compris de la tenue, ont en effet eu vent de la sortie dudit Genzoudi, mais hésitent un peu sur la probabilité qu'il soit parmi les perturbateurs du jour. Finalement, Jean se convainc que Genzoudi est sans doute parmi les jeunes et tous les fonctionnaires BAC présents embarquent dans deux véhicules, en direction de Balzac.

Journal de terrain, 27 mai 2004.

Que retenir de ce moment de délibération collective, visant à déterminer si les « perturbateurs » appelaient l'intervention de la BAC ou seulement celle de la police en tenue? Autrement dit: l'intervention de la police ordinaire, indifférenciée, encadrée par normes policières communes, ou bien « les BAC », (auto-) dotés de leurs prérogatives particulières, de leur style bien à eux, et d'abord de cette supériorité singulière qui leur confère le pouvoir de décider de « choisir » leurs interventions. Le territoire ne détermine pas en lui seul le style de l'opération de police. C'est bien plutôt la probabilité de la présence de Genzoudi, c'est-à-dire d'une personne dotée de sa qualité indubitable de "propriété de police», qui est l'opérateur de qualification du territoire, devenu alors seulement territoire policier, territoire sur lequel les policiers pourront estimer qu'ils jouissent d'une relative souveraineté dans la définition des personnes et des actes à leur opposer ${ }^{32}$.

A partir du moment où le territoire, par l'opérateur de la qualité pénale de la personne, est devenu territoire policier, l'intervention prend une tournure toute particulière.

Arrivés à Balzac, nous sommes salués par un jeune Maghrébin de l'autre côté de la rue, nonchalamment accoudé à sa voiture. Jean lui demande comment il est arrivé jusqu'ici [il est sous le coup d'une suspension de permis] et le jeune lui répond qu'un copain l'a déposé. Jean lui dit de dégager, sauf à vouloir passer une nuit au poste. Vingt mètres plus loin, nous sommes au pied de la Tour Balzac. S'y trouvent 10 jeunes, dont 2 Noirs, 2 ou 3 Blancs et 6 ou 7 Maghrébins, parmi lesquels l'attendu Genzoudi. Jean fait un ample mouvement du bras à tous les jeunes et leur annonce qu'ils ne sont que de la vermine, des parasites et qu'ils passent leur temps à emmerder le monde. Il va chercher Genzoudi, resté assis dans la voiture toutes portes ouvertes, autoradio branchée, et l'insulte tout particulièrement. Il demande ensuite aux jeunes de se mettre «comme d'habitude, les

\footnotetext{
${ }^{32}$ Sur cette notion de souveraineté policière, je me permets de renvoyer à mon ouvrage Bavures policières ?, op. cit., ainsi qu'à « Le nouveau mandat policier. Faire la police dans les zones dites 'de non-droit' », Criminologie, 38, 2, 2005, p. 103-121 (où l'on retrouve Alain, dans une situation cette fois de retenue d'usage de la force).
} 
mains sur le muret, retournés face au mur, contenu des poches sur le muret». Tout le monde est passé au contrôle, les identités sont relevées. Les deux collègues d'Alain et Jean restent silencieux, et Jean ne cesse de dire son mépris aux jeunes contrôlés. Ces derniers restent absolument stoïques, impavides. Alain et Jean entreprennent alors de vider les bouteilles de bière que les jeunes avaient achetées en pack, et envisagent de les briser sur le rebord métallique des poubelles, près duquel je me trouve, un peu en retrait de la ligne de front. Les policiers hésitent à briser les bouteilles et je leur suggère alors de les décapsuler au briquet. Ils me confient le soin de le faire et les bouteilles sont ainsi vidées une à une devant les jeunes dont les visages prennent alors une expression de colère rentrée. Genzoudi proteste : il est « chez lui », ne « dérange personne » et, surtout, n'a pas commis les infractions qu'on lui impute d'un ton véhément (notamment être un voleur éprouvé). Alain tape alors légèrement dans leur ballon de foot, qui commence sa course sur la rue en pente: Genzoudi s'engage derrière le ballon pour le stopper, mais Alain le repousse brutalement. Genzoudi lui déclare alors qu'il fait son boulot salement, et Alain le flanque contre le mur assez violemment, choc que Genzoudi absorbe avec un calme absolu. Genzoudi : «Et bien sûr, c'est parce que je suis un Arabe que vous dites que je suis un voleur», et Alain lui répond droit dans les yeux "Parfaitement! Je suis raciste, et alors?». Fin de l'opération: tous les résidents sont appelés à dégager le terrain. Les jeunes s'exécutent, visiblement très énervés de l'humiliation subie, mais Genzoudi est appelé à mieux garer son véhicule, à cheval sur deux emplacements. Les policiers rigolent lorsqu'ils le voient mettre sa ceinture pour exécuter son créneau. En descendant de la voiture, Genzoudi se tourne à nouveau vers Alain et lui dit qu'il peut tolérer certaines remarques de la part de Florent, ayant déjà eu affaire à lui, mais qu'en ce qui le concerne, il ne peut que constater qu'Alain s'amuse ridiculement à « jouer au cowboy ». Les copains de Genzoudi, déjà éloignés au bas de la rue, l'appellent à « laisser pisser ». Nous retournons aux véhicules, et l'un des policiers, en se tournant à moitié vers moi, peut-être un peu gêné, blague sur cette "nouvelle grande opération de police » [l'une des expressions courantes d'auto-dérision chez les agents].

L'intervention illustre cette logique de basculement dans un mode raciste et vexatoire, humiliant. L’appartenance raciale n'a pas été à elle seule l'opérateur de changement de répertoire. C'est la superposition du profil pénal (et de la familiarité avec au moins un des intervenants policiers) et du territoire, qui ont offert le cadre de possibilité de l'événement et opéré la conversion des attitudes racistes en comportements discriminatoires, dont on imagine sans peine les effets délétères sur les perceptions de la police chez ces jeunes gens de 25-30 ans rassemblés ce soir-là au pied de la tour pour boire une bière en écoutant leur autoradio.

En France, la survenue d'événements tragiques, dont l'histoire récente ne manque pas, ne conduit jamais à la constitution de grandes commissions d'enquête telles que celles que l'on a vu se constituer en Grande-Bretagne et dont l'impact sur les réformes 
policières fut considérable $\mathrm{e}^{33}$. On ne saurait pourtant prendre appui sur cette carence pour imputer à l'institution policière un caractère immuablement raciste et discriminatoire, comme bon nombre de commentateurs l'ont fait à la suite des émeutes de 2005. Au contraire, l'accès au droit des populations les plus exposées (d'abord facilité par le fait que, à la différence de leurs parents, elles disposent de la nationalité française) et la surveillance renforcée des activités policières depuis une quinzaine d'années, en partie sous l'effet d'émeutes liées à des interventions policières mortelles ${ }^{34}$, ont certainement conduit à une raréfaction des marques les plus spectaculaires, les plus évidentes ou les plus brutales de racisme policier.

Cela étant, la permanence de pratiques discriminatoires est attestée. Mais elle l'est sous des formes aujourd'hui souvent plus complexes, plus subtiles que le simple déchaînement d'actes brutaux ou humiliants à caractère racistes, même si ceux-ci, comme nous l'avons noté, ne relèvent pas toujours d'un passé révolu. Nous avons vu que les différences de traitement par les policiers des populations qu'ils rencontrent superposent aux critères ethniques des critères à la fois territoriaux et strictement pénaux. La «clientèle» policière, celle dont les policiers estiment qu'elle leur appartient de fait, et dont ils s'estiment fondés à les traiter différemment, est constituée d'une population dont l'appartenance ethnique n'est pas le seul opérateur de réversibilité, celui qui fait basculer la nature de l'interaction dans l'ordre d'un traitement différencié, brutal ou humiliant.

Cette considération nous oblige à porter un regard prudent sur les indicateurs statistiques. Ainsi, si l'on compare les prévenus du groupe «nord-africain » de notre enquête sur les atteintes à agents de la force publique et les prévenus «sans profession » de l'enquête de Bruno Aubusson de Cavarlay sur les décisions judiciaires

\footnotetext{
${ }^{33}$ Nous pensons en particulier à la commission de Lord Scarman (1982) à la suite des émeutes de Brixton de l'année précédente et plus récemment à la commission McPherson (1999) à la suite de l'enquête calamiteuse menée par les policiers sur un crime raciste commis en 1993.

${ }^{34}$ La présence du médecin au début et de l'avocat à la $23^{\mathrm{e}}$ heure de garde à vue, innovations considérables, a par exemple été introduite par réforme de la procédure pénale prise en 1993 dans le ressac des violences de Mantes la Jolie (1991). La perméabilité des institutions régaliennes françaises au droit européen a également considérablement accru les contraintes pesant sur les policiers, notamment du fait des deux mesures introduites en 2000, à la suite de la condamnation de la France pour torture (1999) : loi sur la présomption d'innocence (présence de l'avocat dès la $1^{\mathrm{e}}$ heure de garde à vue), loi créant la CNDS.
} 
Didier Fassin, Eric Fassin (dir.), De la question sociale à la question raciale ?Représenter la société française. Paris : La Découverte, 2006, p. 211-229.

rendues $^{35}$, on observe que les réactions aux variables explicatives sont très semblables, en ce sens qu'elles laissent toutes deux apparaitre une «clientèle judiciaire », qui rassemble tous ces prévenus toujours plus systématiquement condamnés que les autres, et ce toutes choses égales par ailleurs (y compris l'infraction elle-même), ces prévenus systématiquement punis, donc, avant les actes pour lesquels ils comparaissent. Variables ethniques et variables sociales se superposent ainsi sans nul doute lorsqu'il s'agit de rendre compte des clientèles discriminées par le système pénal et l'intervention policière.

Reste un problème plus redoutable encore: quelle réponse opposer aux discriminations produites par le système pénal, dont on a vu que (au moins à la phase $\mathrm{du}$ jugement) elles n'étaient apparemment pas le fruit de volontés discriminatoires, mais d'un empilement de décisions techniques, qui frappent indifféremment les Blancs et les autres, et condamnent moins les Blancs que les autres. Le problème est en effet redoutable, car il exige d'abord une traçabilité exacte des décisions pénales et de leurs effets toutes choses égales par ailleurs, puis des procédures de redressement des décisions judiciaires qui contredisent à la fois l'indépendance de la justice et le principe d'individualisation de la peine.

Peu de systèmes pénaux se sont attaqués au problème. Un Américain noir condamné à la peine de mort dans l'État de Géorgie pour avoir tué une personne blanche avait récemment introduit un recours à la Cour suprême, arguant qu'il était victime de discrimination dès lors que, toutes choses égales par ailleurs, le risque de sentence capitale dans son Etat était bien plus élevé lorsque le prévenu est un Noir ayant tué un Blanc que dans tout autre cas de figure. La Cour suprême a en effet admis qu'existe une discrimination statistique (McCleskey c/ Kemp, 1997), mais a rejeté le recours du condamné, arguant qu'il lui fallait faire la preuve (qu'il avait en l'espèce), qu'au cours du procès qui l'avait condamné, il avait été victime de décisions biaisées par sa couleur de peau.

Michael Tonry en retient l'apologue suivant : si un Martien débarquait aux États-

\footnotetext{
${ }^{35}$ Celle où, à partir des 342000 condamnations prononcées en contradictoire durant l'année 1978, l'auteur concluait par: "Veut-on caricaturer? L'amende est bourgeoise et petite-bourgeoise, l'emprisonnement ferme est sous-prolétarien, l'emprisonnement avec sursis est populaire» (« Hommes, peines et infractions », op. cit., p. 275-309).
} 
Didier Fassin, Eric Fassin (dir.), De la question sociale à la question raciale ?Représenter la société française. Paris : La Découverte, 2006, p. 211-229.

Unis, il constaterait qu'une entreprise employant un taux de minorités inférieur à leur représentation dans la population locale s'expose à un risque certain de condamnation par la justice pénale. L'institution judiciaire ne s'expose quant à elle à rien du tout, alors même que la surreprésentation des Noirs qu'elle fait exécuter est patente; comme s'il importait plus que soit respectée l'égalité face à l'emploi que face à la peine capitale ${ }^{36}$. On pourrait imaginer, en France, des mécanismes correcteurs des effets empilés des décisions pénales lorsqu'elles portent préjudice à des groupes sociaux ou à des groupes ethniques. De tels instruments ébranleraient assurément jusqu'aux fondations mêmes de la justice pénale.

\footnotetext{
${ }^{36}$ Michael TONRY, Thinking about crime, op. cit., p. 223.
} 